\title{
ANALYSIS OF FLIGHT MANAGEMENT SYSTEM PREDICTIONS OF IDLE-THRUST DESCENTS
}

\author{
Laurel Stell, NASA Ames Research Center, Moffett Field, CA
}

\begin{abstract}
To enable arriving aircraft to fly optimized descents computed by the flight management system (FMS) in congested airspace, ground automation must accurately predict descent trajectories. To support development of the predictor and its uncertainty models, descents from cruise to the meter fix were executed using vertical navigation in a B737-700 simulator and a B777-200 simulator, both with commercial FMSs. For both aircraft types, the FMS computed the intended descent path for a specified speed profile assuming idle thrust after top of descent (TOD), and then it controlled the avionics without human intervention. The test matrix varied aircraft weight, descent speed, and wind conditions. The first analysis in this paper determined the effect of the test matrix parameters on the FMS computation of TOD location, and it compared the results to those for the current ground predictor in the Efficient Descent Advisor (EDA). The second analysis was similar but considered the time to fly a specified distance to the meter fix. The effects of the test matrix variables together with the accuracy requirements for the predictor will determine the allowable error for the predictor inputs. For the B737, the EDA prediction of meter fix crossing time agreed well with the FMS; but its prediction of TOD location probably was not sufficiently accurate to enable idle-thrust descents in congested airspace, even though the FMS and EDA gave similar shapes for TOD location as a function of the test matrix variables. For the B777, the FMS and EDA gave different shapes for the TOD location function, and the EDA prediction of the TOD location is not accurate enough to fully enable the concept. Furthermore, the differences between the FMS and EDA predictions of meter fix crossing time for the B777 indicated that at least one of them was not sufficiently accurate.
\end{abstract}

\section{Introduction}

In congested airspace today, controllers direct aircraft to descend in steps. Since air density, and hence drag, increase as the aircraft descends, significant reductions in fuel consumption and emissions would result if aircraft stayed at cruise altitude longer and then descended smoothly at idle thrust. The flight management system (FMS) on a large jet can compute the location of top of descent (TOD) assuming an idle-thrust descent. To merge aircraft, however, controllers impose level flight segments, which make it much easier for them to estimate the relative speeds of two aircraft given their calibrated airspeed (CAS). ThreeDimensional Path Arrival Management (3DPAM) enables arriving aircraft to fly optimized descents computed by the FMS in congested airspace [1]. The 3DPAM ground automation must accurately predict descent trajectories to ensure there are no traffic conflicts and to provide situation awareness to controllers. In addition to predicting the meter fix arrival time accurately to ensure lateral separation, accurate prediction of the vertical profile is essential to ensure vertical separation from aircraft at different altitudes, including crossing traffic.

In the 3DPAM concept, the FMS will be given the descent speed profile and the speed and altitude at the unknown TOD location and at the meter fix. Assuming idle thrust in the descent, the FMS will compute the vertical profile and fly it with minimal human intervention. In this study, such descents were flown in a simulator with a commercial FMS and no human intervention. The test matrix varied aircraft weight, descent speed, and wind conditions to determine their effect on FMS prediction in a B737-700 simulator and a B777200 simulator.

Due to the potential fuel savings and emissions reductions, enabling continuous descents is being pursued by several research groups $[2,3,4,5,6]$. Most of the previous error analysis has focused on arrival time at a waypoint. A few, such as [2,5], have also considered the operational TOD prediction error. Using a simulator in the current research made it possible to use a test matrix, which simplified analyzing and visualizing the effects of individual factors. Tong, Boyle, and Warren [3] and Herndon et al. [7,8] also used simulators with commercial FMSs but analyzed the effects of different factors; the former considered the de-icing setting (which is probably also important for 3DPAM) and the latter emphasized comparing aircraft types.

The first analysis reported in this paper deter- 
mined the effect of the test matrix parameters on TOD location computed by both the FMS and the Efficient Descent Advisor (EDA), which is the proposed predictor for 3DPAM. The polynomial approximation approach was very similar to a previous paper by the same author [9]. Analysis of actual TOD locations extracted from operational data [10] indicated that the TOD path distance can be approximated well by a linear function in cruise and descent altitudes, descent CAS, wind, and aircraft weight. This prompted a second look at the FMS predictions of TOD location, focusing this time on models linear in descent speed and aircraft weight. To better investigate the applicability of a linear model to multiple aircraft types, the B777 data previously omitted were included this time. Furthermore, this paper includes results for predictions from EDA, whereas the previous paper only analyzed the FMS-computed TOD locations. To interpret what is significant variation, previous controller feedback suggests that acceptable ground automation TOD prediction error is likely to be no larger than $5 \mathrm{nmi}$ in order to enable optimized descents in congested airspace.

The second analysis in this paper was similar to the first but considered the time to fly a specified distance to the meter fix. To interpret these results, consider that the minimum separation requirement at the meter fix is $5 \mathrm{nmi}$, which is roughly $60 \mathrm{sec}$ in zero wind at the meter fix speed and altitude constraints. Consequently, the ground automation probably needs to predict the meter fix arrival time within about $20 \mathrm{sec}$.

Since the FMS-computed TOD location should be within a few miles of its actual location, comparison of the EDA and FMS predictions of TOD location should give a reasonable estimate of the operational accuracy of EDA, but the data have the advantage of being much less noisy than operational data. The FMS prediction of the meter fix crossing time, on the other hand, may be significantly different from the actual time due to failure to maintain the target descent speed or due to error in the wind conditions entered into the FMS. Comparison of the EDA and FMS predictions of these times can still be useful, however, in identifying significant differences between the predictors. Furthermore, the accuracy of both predictions will be important in the future when data link is used. From both analyses, the effects of the test matrix variables will help determine the allowable error for the predictor inputs.

\section{Experiment Procedures}

The experiments in this study were run in simulators - a B737-700 and a B777-200 — operated by Boeing Phantom Works. These simulators were custom-built and each included a commercial FMS, which had different manufacturers for these two aircraft types. These simulators were designed for developing procedures, not pilot training. They include random noise. The FMS used both lateral and vertical navigation.

To compute its descent trajectory, the FMS used the constraints shown in Figure 1, which uniquely determined the trajectory. The parameters shown in each box were specified for that segment or that point of the descent. In brief, the FMS assumed idle thrust from TOD to the meter fix, and it used the speed profile, which will be specified in 3DPAM by the controller for both cruise and descent. The FMS also used two meter fix constraints: altitude $11,000 \mathrm{ft}$ and speed $250 \mathrm{kt} \mathrm{CAS}$ (KCAS). In Figure 1, the first segment in the descent had constant Mach number, which was the same as the cruise Mach number. As the altitude decreased, the CAS increased until it reached the target descent CAS, which will be specified by the controller in 3DPAM. The next segment was then flown at that CAS. The final segment only existed if the descent CAS was faster than the meter fix speed constraint, in which case the aircraft pitched up to decelerate to that constraint. The preceding assumed descent CAS was faster than the cruise CAS. If this was not the case, the aircraft first decelerated at idle thrust while maintaining level flight at cruise altitude or close to it, and then it pitched down to maintain the target descent speed at idle thrust.

The horizontal trajectory was the same nearlystraight standard arrival route in all simulation runs. Each run started at least $100 \mathrm{nmi}$ from the meter fix in cruise at $37,000 \mathrm{ft}$. To set up the descent in the FMS in these experiments, the waypoints were entered, "ECON MODE" was selected to specify an idlethrust descent, and the FMS-computed descent speed was overridden by manually entering the desired descent speed, which will be specified by the controller in 3DPAM. The cruise Mach number was determined by the FMS based on a user-specified parameter, but it was generally between 0.79 and 0.80 . The simulation runs continued below the meter fix, but analysis included only data to the meter fix.

Two different sets of conditions were used, shown in Table 2 and Table 3 . The former describes nine runs, while the latter describes 22 runs. Five of the entries in the two sets were duplicates and run twice. The values of the aircraft weight depended upon aircraft type as shown in Table 1. 


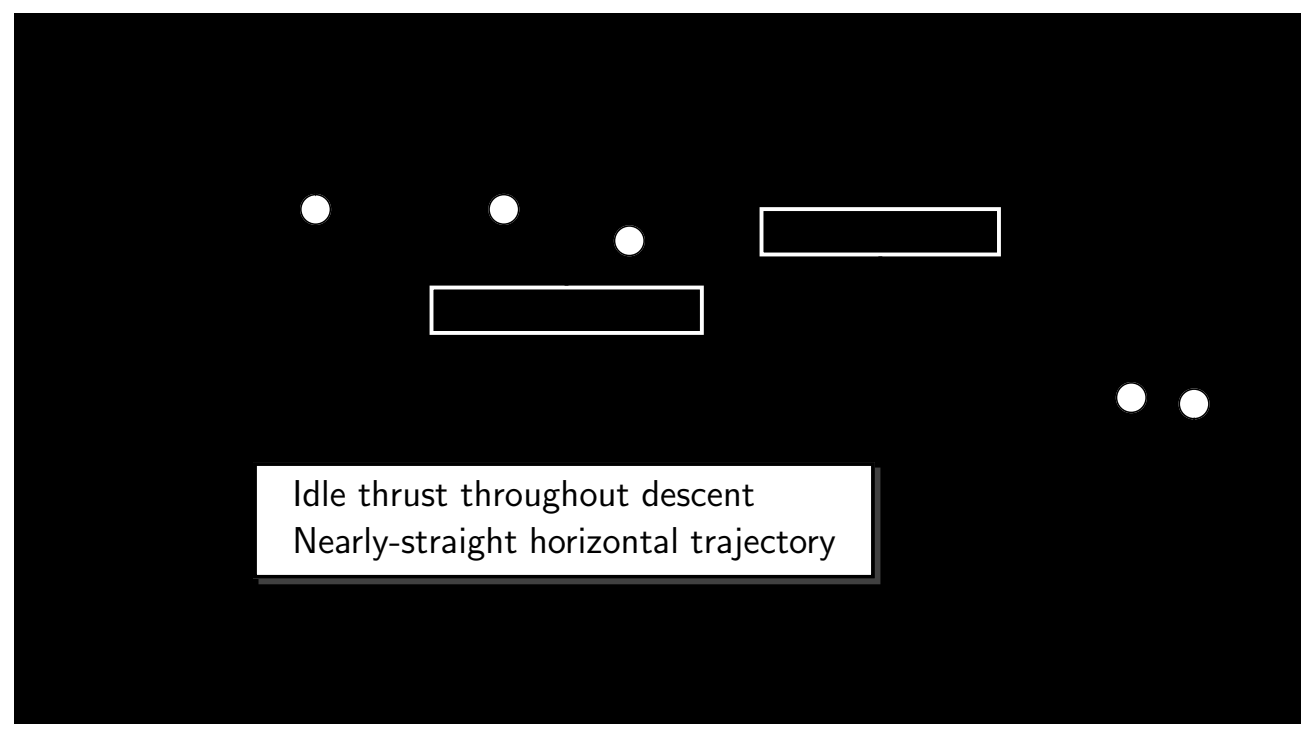

Figure 1. Idle-thrust Descent Schematic

Table 1. Test Matrix Aircraft Weights

\begin{tabular}{l|rc} 
& \multicolumn{1}{|c}{ B737 } & B777 \\
\hline LIGHT_WT & $92,000 \mathrm{lb}$ & $312,000 \mathrm{lb}$ \\
NOMINAL_WT & $117,000 \mathrm{lb}$ & $379,000 \mathrm{lb}$ \\
HEAVY_WT & $131,200 \mathrm{lb}$ & $447,000 \mathrm{lb}$
\end{tabular}

Table 2. First Set of Test Conditions

\begin{tabular}{c|c|c}
\multicolumn{3}{c}{ Zero Winds in All Nine Runs } \\
\hline \hline 250 KCAS & 250 KCAS & 250 KCAS \\
LIGHT_WT & NOMINAL_WT & HEAVY_WT \\
\hline 280 KCAS & 280 KCAS & 280 KCAS \\
LIGHT_WT & NOMINAL_WT & HEAVY_WT \\
\hline 320 KCAS & 320 KCAS & 320 KCAS \\
LIGHT_WT & NOMINAL_WT & HEAVY_WT
\end{tabular}

All the trajectories are described by the schematic in Figure 1 except those with descent speed 250 KCAS, which is slower than the cruise speed. The test conditions were chosen to cover most of the expected operating conditions for descent speed and aircraft weight. The distribution of winds aloft and their forecast errors in the operational environment were unknown, so they were chosen somewhat arbitrarily. Since the horizontal trajectory is nearly straight, the wind fields used were actually constant in space and time with direction nearly parallel to the arrival route. The wind conditions labeled "in FMS" means the data entered into the descent wind page of the FMS.

\section{Data}

The analysis of FMS predictions primarily used Automatic Dependent Surveillance-Contract (ADS-C) messages that Boeing provided. The time between ADS-C messages generally did not exceed $64 \mathrm{sec}$, but sometimes it was almost $200 \mathrm{sec}$. The only ADS-C data used in this analysis were the current position and the FMS trajectory predictions, which are in the part of the message labeled Intermediate Projected Intent Group (IPIG). Each IPIG contains the distance and true track from the previous group or from the current position for the first group, the predicted barometric altitude, and the predicted time to reach the point from the current position. The path distance of each prediction point is computed for analysis by projecting the initial position onto the arrival route, computing its path distance relative to the meter fix, and adding the given distances between waypoints.

In analysis of the FMS-computed TOD location, only the last message sent before the aircraft descended from cruise altitude was used. The IPIGs in this message include both the FMS-computed TOD and the meter fix. In analysis of the FMS-computed time $T_{f}$ to fly distance $D_{f}$ to the meter fix, only the last message sent at least distance $D_{f}$ before the meter fix was used. Since this prediction rarely starts exactly distance $D_{f}$ from the meter fix, linear interpolation was used to estimate $T_{f}$. 
Table 3. Second Set of Test Conditions

\begin{tabular}{|c|c|}
\hline Subset A & Subset B \\
\hline $\begin{array}{l}117,000 \mathrm{lb}, \\
\text { Zero Winds, } \\
\text { and One }\end{array}$ & $\begin{array}{l}280 \text { KCAS, } \\
\text { Zero Winds, } \\
\text { and One }\end{array}$ \\
\hline Speed Below & Weight Below \\
\hline $\begin{array}{l}250 \mathrm{KCAS} \\
260 \mathrm{KCAS} \\
270 \mathrm{KCAS} \\
280 \mathrm{KCAS} \\
290 \mathrm{KCAS} \\
300 \mathrm{KCAS} \\
310 \mathrm{KCAS} \\
320 \mathrm{KCAS}\end{array}$ & $\begin{array}{c}92,000 \mathrm{lb} \\
131,200 \mathrm{lb}\end{array}$ \\
\hline
\end{tabular}

When creating the EDA predictions used in this analysis, the appropriate aircraft weight in Table 1 was used, even though EDA typically uses the same weight for each aircraft type. The cruise Mach number is estimated by the mean of the recorded Mach number in cruise from the simulator state data. The initial condition for each prediction is at a point with path distance $211 \mathrm{nmi}$ from the meter fix. For the descent rate for the final segment before the meter fix in Figure 1, the data did not indicate an obvious constraint in terms of vertical speed, descent rate in $\mathrm{ft} / \mathrm{nmi}$, or flight path angle. Therefore, the EDA predictor simply decelerates at level flight, which moves TOD closer to the meter fix than it would be if the segment were not level but most likely by less than $1 \mathrm{nmi}$.

\section{Analysis of B737 Data}

The rest of this paper discusses the results of the data analysis. To make the story easier to follow, this section presents all the results for the B737, and the next section presents all the results for the B777. The details of the analysis are presented in this section along with the results, whereas the section on the B777 focuses on the results.

\author{
Subset C \\ $280 \mathrm{KCAS}$, \\ $117,000 \mathrm{lb}$, \\ and One Set of \\ Wind Conditions Below \\ $25 \mathrm{kt}$ Tailwind in Simulator and FMS \\ $50 \mathrm{kt}$ Tailwind in Simulator and FMS \\ $25 \mathrm{kt}$ Headwind in Simulator and FMS \\ $50 \mathrm{kt}$ Headwind in Simulator and FMS \\ $25 \mathrm{kt}$ Tailwind in Simulator, Zero Winds in FMS \\ $50 \mathrm{kt}$ Tailwind in Simulator, Zero Winds in FMS \\ $25 \mathrm{kt}$ Headwind in Simulator, Zero Winds in FMS \\ $50 \mathrm{kt}$ Headwind in Simulator, Zero Winds in FMS \\ Zero Winds in Simulator, 25 kt Tailwind in FMS \\ Zero Winds in Simulator, 50 kt Tailwind in FMS \\ Zero Winds in Simulator, $25 \mathrm{kt}$ Headwind in FMS \\ Zero Winds in Simulator, $50 \mathrm{kt}$ Headwind in FMS
}

\section{TOD Location}

In this section, the separate effects of descent speed, weight, and wind on TOD location are investigated. The FMS prediction of the TOD location is critical because, as long as the FMS flies the descent, the actual TOD will be close to the FMS prediction. Therefore, even if the ground automation trajectory predictor has more accurate inputs or models than does the FMS, the ground prediction of TOD will rarely be closer to the actual TOD than is the FMS prediction. Furthermore, the FMS predictions of TOD location in these experiments are the same as they would have been in an operational environment. To analyze the TOD location, the trajectory is viewed as two-dimensional, with horizontal location specified as distance along the path relative to the meter fix, which means the TOD location is then a scalar.

\section{Effect of Descent Speed and Aircraft Weight}

To quantify the effect of descent speed and weight on TOD, the TOD path distance for the runs with zero wind (all the runs except those in Subset $\mathrm{C}$ of Table 3) was approximated by least squares fit to a linear polynomial. The markers in Figure 2 show the FMScomputed values of TOD path distance, and the lines show the values of this approximation for the weights 

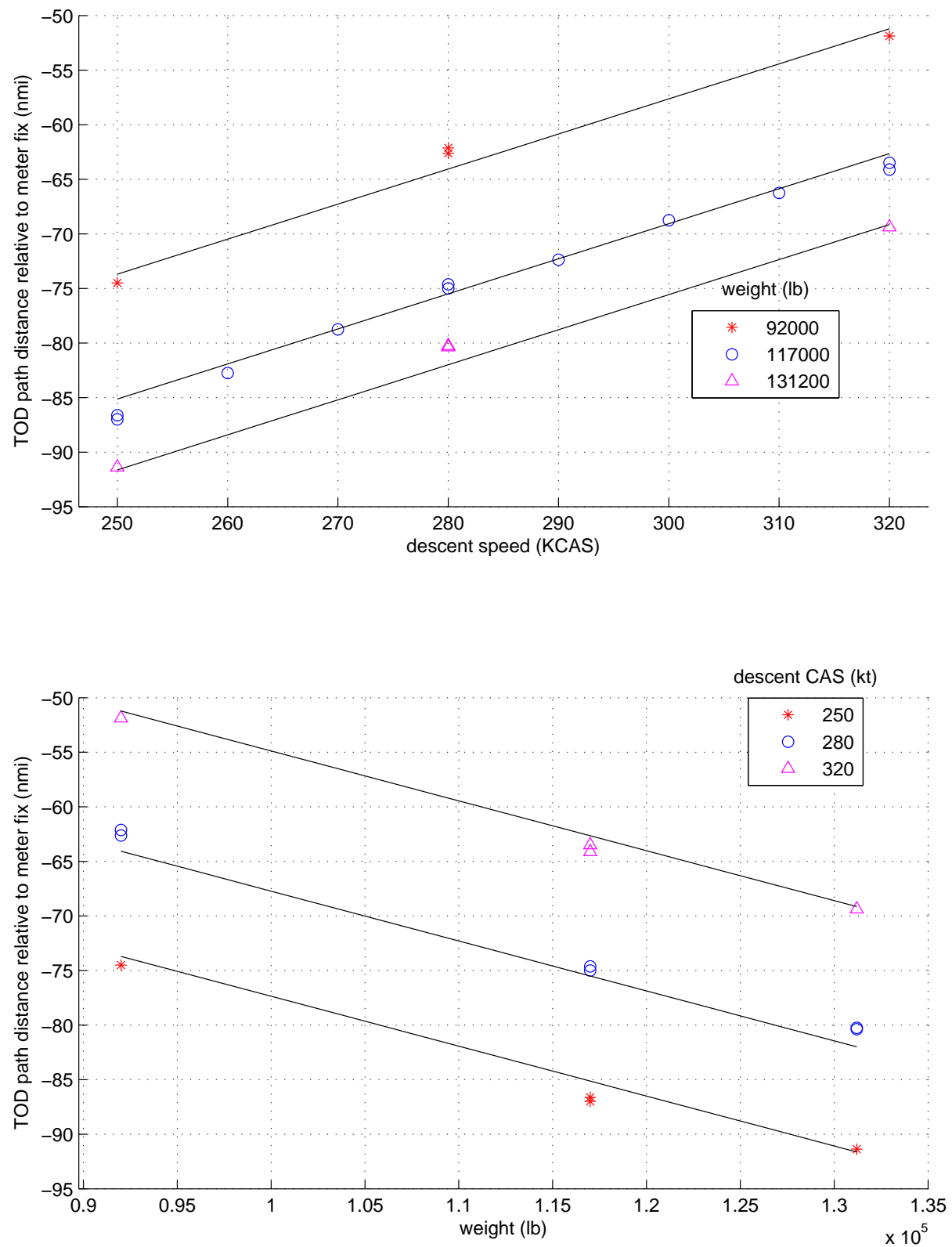

Figure 2. Speed, Weight Effect on B737 FMS TOD

and descent speeds in these runs. Table 4 shows the coefficients and goodness-of-fit statistics of this approximation. The coefficients also indicate the following:

- For each 10,000 $\mathrm{lb}$ increase in weight, TOD is about $4.6 \mathrm{nmi}$ farther from the meter fix.

- Increasing the descent speed by $10 \mathrm{KCAS}$ moves TOD about $3.2 \mathrm{nmi}$ closer to the meter fix.

Assuming the operational ranges of weight and descent speed would be close to their ranges in the test ma- trix, using a nominal value for either weight or descent speed would result in errors exceeding $5 \mathrm{nmi}$ even if the predictor were otherwise perfect.

The next question is whether the TOD locations predicted by EDA exhibit the same behavior. The plots analogous to Figure 2 look essentially the same, but the EDA TOD tends to be about $4 \mathrm{nmi}$ closer than the FMS value to the meter fix. This is reflected in the differences in the coefficients in Table 4. These results confirm that the TOD location determined by the equa- 
Table 4. Speed, Weight Coefficients in TOD Linear Approximation

\begin{tabular}{l|rr|rr}
\multirow{2}{*}{ Term } & \multicolumn{2}{|c|}{ B737 } & \multicolumn{2}{c}{ B777 } \\
\cline { 2 - 5 } & \multicolumn{1}{|c}{ FMS } & \multicolumn{1}{c}{ TS } & \multicolumn{1}{c}{ FMS } & \multicolumn{1}{c}{ TS } \\
\hline \hline Constant & -110 & -110 & -120 & -170 \\
Descent CAS (kt) & 0.32 & 0.30 & 0.29 & 0.39 \\
Weight (lbs) & -0.00046 & -0.00039 & -0.00013 & -0.00012 \\
\hline$R^{2}$ value & 0.988 & 0.990 & 0.990 & 0.969 \\
RMS residual & $1.1 \mathrm{nmi}$ & $0.92 \mathrm{nmi}$ & $0.92 \mathrm{nmi}$ & $2.0 \mathrm{nmi}$ \\
max abs residual & $1.9 \mathrm{nmi}$ & $1.6 \mathrm{nmi}$ & $1.8 \mathrm{nmi}$ & $5.6 \mathrm{nmi}$
\end{tabular}

tions of motion can be essentially linear in descent speed and aircraft weight over their ranges in the test matrix used here.

\section{Effect of Wind}

To characterize the effect of wind on TOD location, first consider the six simulator runs in Subset $\mathrm{C}$ of Table 3 with the same winds used by the FMS and the simulator. The circles in Figure 3 show TOD path distance for these runs, and the line shows that this distance is linear in the wind, with a $25-\mathrm{kt}$ increase in the wind speed moving the TOD location about $4.7 \mathrm{nmi}$ farther from the meter fix.

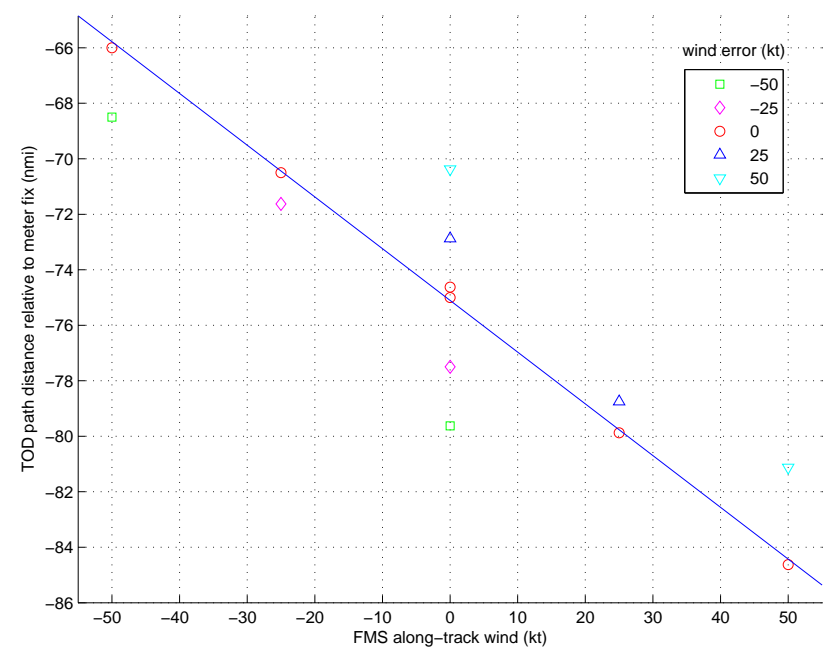

Figure 3. Effect of Wind on B737 FMS TOD

There is a simple explanation for the relationship between TOD and wind. Since the wind in each run is constant over space and time and the speed constraints are given as airspeed, the parameters in the equations of motion relative to the air mass as well as in their constraints are the same in these six runs. Therefore, the flight path angle relative to the air mass is the same function of altitude in these runs, and it is also the same function of time relative to the meter fix time. The change in TOD path distance between any two of these runs is consequently equal to the difference in the distance the air mass moves relative to ground in the time required to descend from TOD to the meter fix. Due to small differences in the cruise speeds between runs, these descent times vary over 690 to $700 \mathrm{sec}$ in these runs. Hence, a change of $25 \mathrm{kt}$ in wind speed should move TOD roughly $(695 \mathrm{sec} \times 25 \mathrm{kt}=4.8 \mathrm{nmi})$, which agrees well with the regression line in Figure 3.

Now consider the runs in Subset $\mathrm{C}$ of Table 3 in which the simulated winds were different than those entered into the FMS descent wind page. These are indicated by the markers other than the circles in Figure 3. The FMS TOD prediction depends upon the error in the winds entered into its descent wind page because the FMS blends that wind field with the current wind measurement, which in these experiments is a simulated pitot tube measurement. The blending algorithm is proprietary, so we cannot explain how the TOD location depends upon wind error.

For comparison, the EDA predictions use only the wind forecast entered into the FMS, not the simulated winds. The TOD locations predicted by EDA fit a line very well. A 25-kt increase in the wind speed moves the TOD location about $4.5 \mathrm{nmi}$, which is slightly smaller than for the FMS-computed value due to shorter descent times - 654 to $658 \mathrm{sec}$ - because the TOD is closer to the meter fix. 


\section{Meter Fix Crossing Time}

To compare the descent times, it is insufficient to consider only the time between TOD and meter fix, since TOD location is not the same in all cases. Instead, we want to compare the time to fly some fixed distance $D_{f}$ to the meter fix. To investigate the effect of the test matrix variables on the entire descent, the value of $D_{f}$ should be larger than the farthest TOD from the meter fix but close enough to the meter fix that the simulator start-up transients have died by that point. For the B737, $D_{f}$ is $95 \mathrm{nmi}$.

The portion of the trajectory under consideration here consists of a cruise segment and a descent segment. For a given simulator run, the ground speed should be essentially constant $-\hat{v}_{c}$, say - over the cruise segment since the Mach number, altitude, and wind conditions are constant except for small random errors. The ground speed will clearly vary over the descent segment, but suppose it has mean value $\hat{v}_{d}$. Then the predicted time $T_{f}$ to fly distance $D_{f}$ to the meter fix can be approximated as

$$
T_{f} \approx \frac{D_{f}+S_{\mathrm{TOD}}}{\hat{v}_{c}}-\frac{S_{\mathrm{TOD}}}{\hat{v}_{d}},
$$

where $S_{\text {TOD }}$ is the TOD path distance relative to the meter fix - wich is negative. This expression shall be further manipulated in the next two subsections to explain the dependence of $T_{f}$ on descent speed, aircraft weight, and wind.

\section{Effect of Descent Speed and Aircraft Weight}

For the trajectories with zero wind, $S_{\mathrm{TOD}}$ can be approximated well by a linear polynomial in descent speed and aircraft weight, and the airspeed is equal to ground speed. Furthermore, for descent speed $v$ ranging over 250 to $300 \mathrm{KCAS}, v^{-1}$ is essentially linear in $v$ with relative error less than $1 \%$. Therefore, if $\hat{v}_{d}$ is approximately proportional to $v$, then substitution into equation (1) and simplification give

$$
T_{f} \approx \gamma_{0}+\gamma_{1} v+\gamma_{2} w+\gamma_{3} v w+\gamma_{4} v^{2}
$$

since $\hat{v}_{c}$ is roughly the same for all the trajectories with zero wind.

Figure 4 and Table 5 essentially confirm this, but they omit the interaction term because it had very little effect. They show not only that this is a good approximation but also that the second-order term in descent speed is significant. For the weights and descent speeds in these runs, the solid lines in Figure 4 show the values of the approximation that is linear in weight and quadratic in descent speed. For each 10,000 lb in-

Table 5. Speed, Weight Coefficients in Fix Time Approximations

\begin{tabular}{l|rr|rr}
\multirow{2}{*}{ Term } & \multicolumn{2}{c|}{ B737 } & \multicolumn{2}{c}{ B777 } \\
\cline { 2 - 5 } & \multicolumn{1}{|c}{ FMS } & \multicolumn{1}{c}{ TS } & FMS & \multicolumn{1}{c}{ TS } \\
\hline \hline \multicolumn{4}{c|}{ linear model } \\
Constant & 1300 & 1300 & 1600 & 1900 \\
Weight (lbs) & 0.00061 & 0.00046 & 0.00010 & $3.0 \times 10^{-5}$ \\
\hline$R^{2}$ value & 0.948 & 0.949 & 0.946 & 0.956 \\
RMS residual & $11 \mathrm{sec}$ & $11 \mathrm{sec}$ & $14 \mathrm{sec}$ & $15 \mathrm{sec}$ \\
max abs residual & $18 \mathrm{sec}$ & $17 \mathrm{sec}$ & $22 \mathrm{sec}$ & $27 \mathrm{sec}$ \\
\hline \hline \multicolumn{1}{c}{ model linear in weight and quadratic in descent speed } \\
\hline Constant & 3000 & 2900 & 3400 & 4000 \\
Descent CAS (kt) & -13 & -13 & -16 & -18 \\
Weight (lbs) & 0.00061 & 0.00046 & 0.00010 & $3.0 \times 10^{-5}$ \\
CAS & 0.020 & 0.020 & 0.023 & 0.026 \\
\hline$R^{2}$ value & 0.995 & 0.997 & 0.994 & 0.996 \\
RMS residual & $3.4 \mathrm{sec}$ & $2.8 \mathrm{sec}$ & $4.6 \mathrm{sec}$ & $4.3 \mathrm{sec}$ \\
max abs residual & $8.1 \mathrm{sec}$ & $7.1 \mathrm{sec}$ & $9.4 \mathrm{sec}$ & $12 \mathrm{sec}$ \\
\hline \hline
\end{tabular}



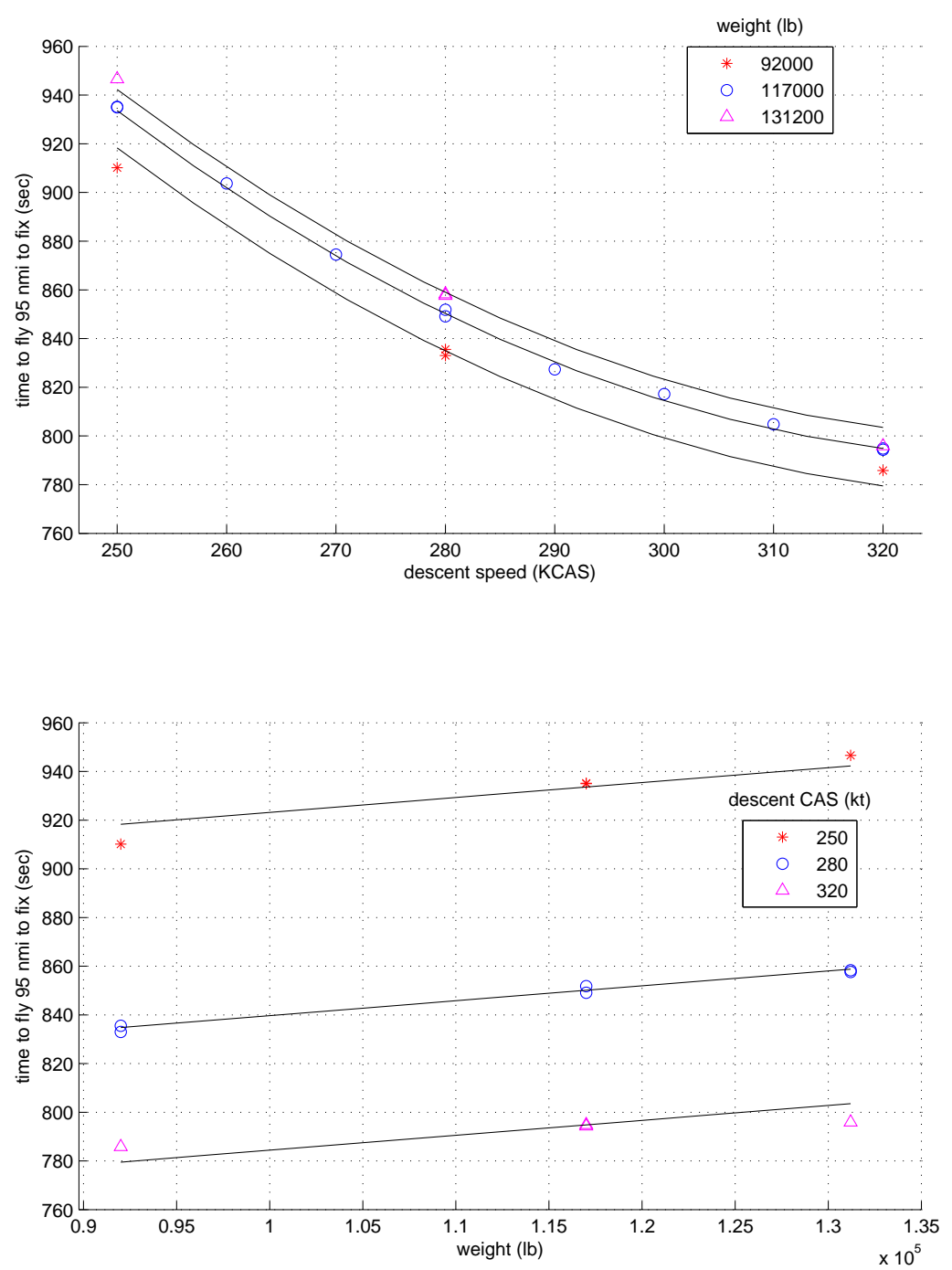

Figure 4. Speed, Weight Effect on B737 FMS Fix Time

crease in weight, $T_{f}$ is about $6.1 \mathrm{sec}$ longer. If the values used in these experiments cover the operational range of weight, then using a nominal value in the middle of this range would cause errors less than $0.5 \times 0.00061 \times(131200-92000)=12 \mathrm{sec}$. Whether this is acceptable would depend upon the error due to other sources.

Again, the analogous plots for the EDA predictions look essentially the same, but EDA predicts $T_{f}$ to be 1 to $10 \mathrm{sec}$ shorter than the FMS does.

\section{Effect of Wind}

To characterize the effect of wind on meter fix crossing time $T_{f}$, first consider the six simulator runs with nominal weight and descent speed and with the same winds used by the FMS and the simulator. The circles in Figure 5 show $T_{f}$ for these runs, and the solid line in this plot shows that this time is approximately linear in wind speed, with a $25-\mathrm{kt}$ increase in wind speed decreasing $T_{f}$ by about $46 \mathrm{sec}$. For these six runs, EDA predicts $T_{f}$ to be 4 to $8 \mathrm{sec}$ shorter than the FMS does.

To see why $T_{f}$ is roughly linear in along-track wind $W_{v}$, first recall that the time between TOD and meter fix is independent of wind. Furthermore, $S_{\mathrm{TOD}}$ is linear in $W_{v}$, and ground speed $\hat{v}_{c}$ in cruise is constant for each descent and equal to $V_{t}+W_{v}$, where $V_{t}$ is roughly the same for all descents. Hence, as in 


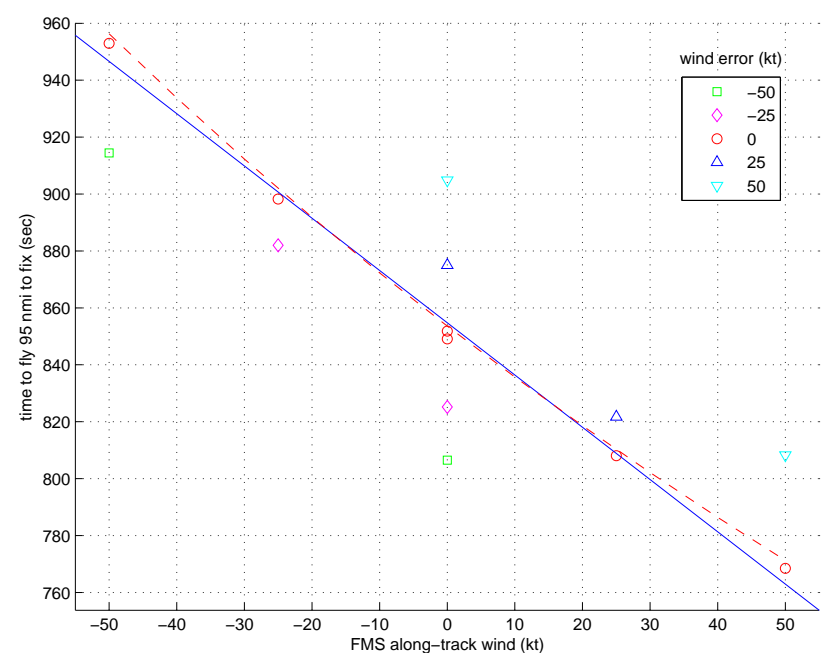

Figure 5. Effect of Wind on B737 FMS Fix Time

equation (1),

$$
T_{f} \approx K+\frac{D_{f}+\left(S_{\mathrm{TOD}}^{(0)}+\alpha W_{v}\right)}{\hat{v}_{c}^{(0)}+W_{v}},
$$

where $S_{\mathrm{TOD}}^{(0)}$ is the TOD path distance for zero wind and $\hat{v}_{c}^{(0)}$ is the cruise ground speed. For the FMS predictions for the B737, $K$ is $695 \mathrm{sec}$ from the above discussion of the effect of wind on TOD location, $S_{\mathrm{TOD}}^{(0)}$ is $-75 \mathrm{nmi}$ and $\alpha$ is $-0.19 \mathrm{hr}$ from Figure 3, and a plot of ground speed for descents with zero wind shows that $\hat{v}_{c}^{(0)}$ is $454 \mathrm{kt}$. The dashed line in Figure 5 shows this approximation, which suggests it is more accurate than the least squares line.

The markers other than the circles in Figure 5 indicate the runs in Subset C of Table 3 in which the simulated winds were different than those entered into the FMS descent wind page. As with the TOD location, the effect of this error is not quantified, but comparison with Figure 3 shows that its effect on $T_{f}$ is roughly proportional to its effect on TOD location.

\section{Analysis of B777 Data}

Now the B777 data is analyzed. The steps in the analysis are the same as for the B737 above.

\section{TOD Location}

The first step is again to investigate the separate effects of descent speed, weight, and wind on TOD location.

\section{Effect of Descent Speed and Aircraft Weight}

Figure 6 shows the effect of descent speed and aircraft weight on the TOD computed by the FMS for the runs in which both the FMS and simulator used zero winds. The lines show the values for a linear approximation, and Table 4 above shows the coefficients and goodness-of-fit statistics. The coefficients indicate the following:

- For each 10,000 $\mathrm{lb}$ increase in weight, TOD is about $1.3 \mathrm{nmi}$ farther from the meter fix.

- Increasing the descent speed by 10 KCAS moves TOD about $2.9 \mathrm{nmi}$ closer to the meter fix.

While the coefficient of aircraft weight for the B777 is significantly less than for the B737, the range of weights is larger. Consequently, the difference in TOD location between the extrema of weight is about the same for the two aircraft types. As with the B737, using a nominal value for either weight or descent speed would result in errors exceeding $5 \mathrm{nmi}$ even if the predictor were otherwise perfect.

The first issue with the B777 data is that the linear approximation of the EDA predictions of TOD location is a significantly worse fit than for the FMS-computed values for the B777 as shown by the solid lines in Figure 7. Of course, the EDA prediction error for the B777 is much worse than for the B737 as shown in Figure 8, which includes all runs except those in Subset C of Table 3. Not only is the absolute value of the median error larger but the variance is also larger. It is not known why TOD location as a function of weight and descent speed has a significantly different shape in this case than it has in the three other cases, but clearly the EDA predictor cannot be used for sensitivity studies in place of a B777 FMS. 

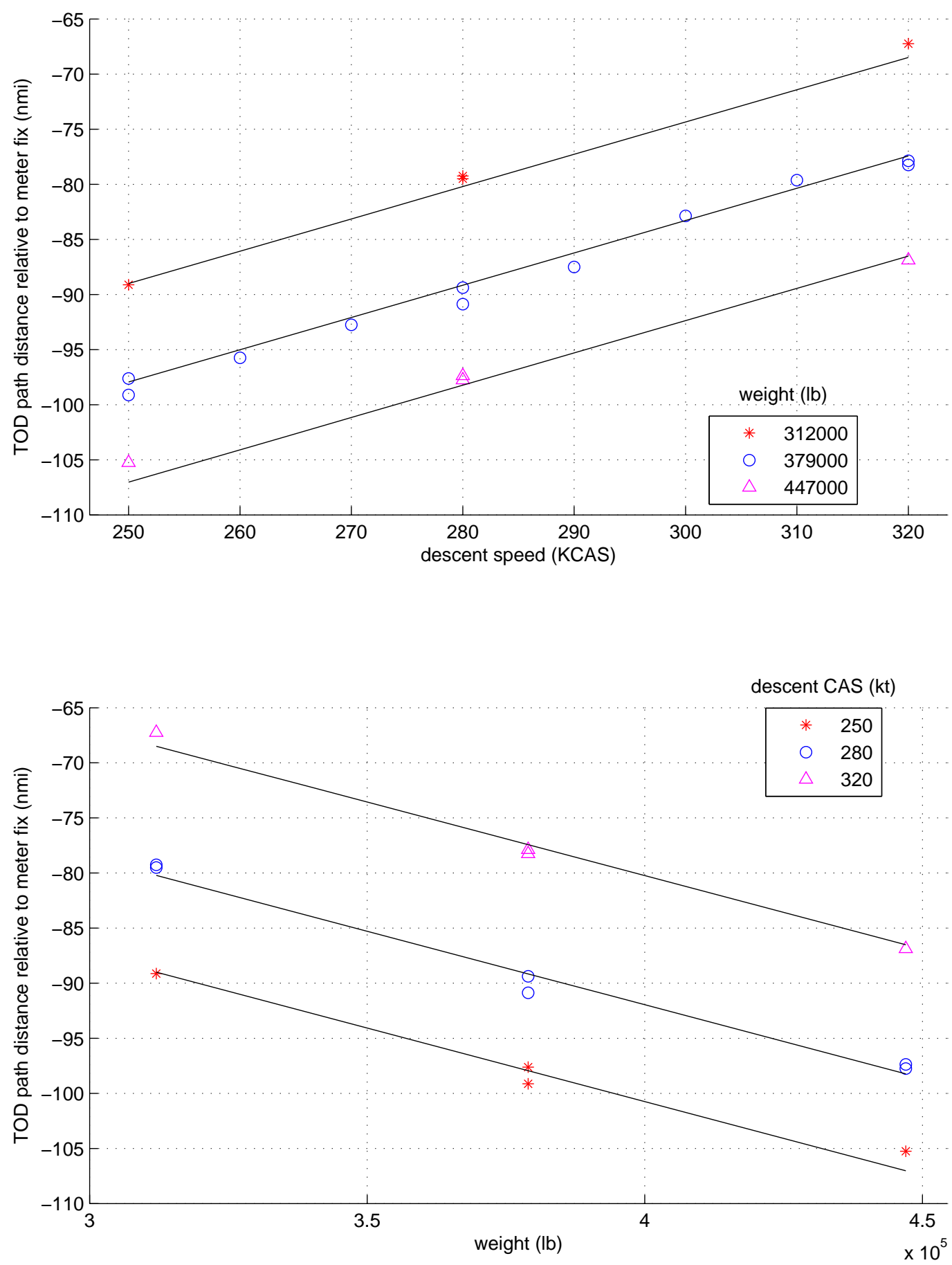

Figure 6. Speed, Weight Effect on B777 FMS TOD 

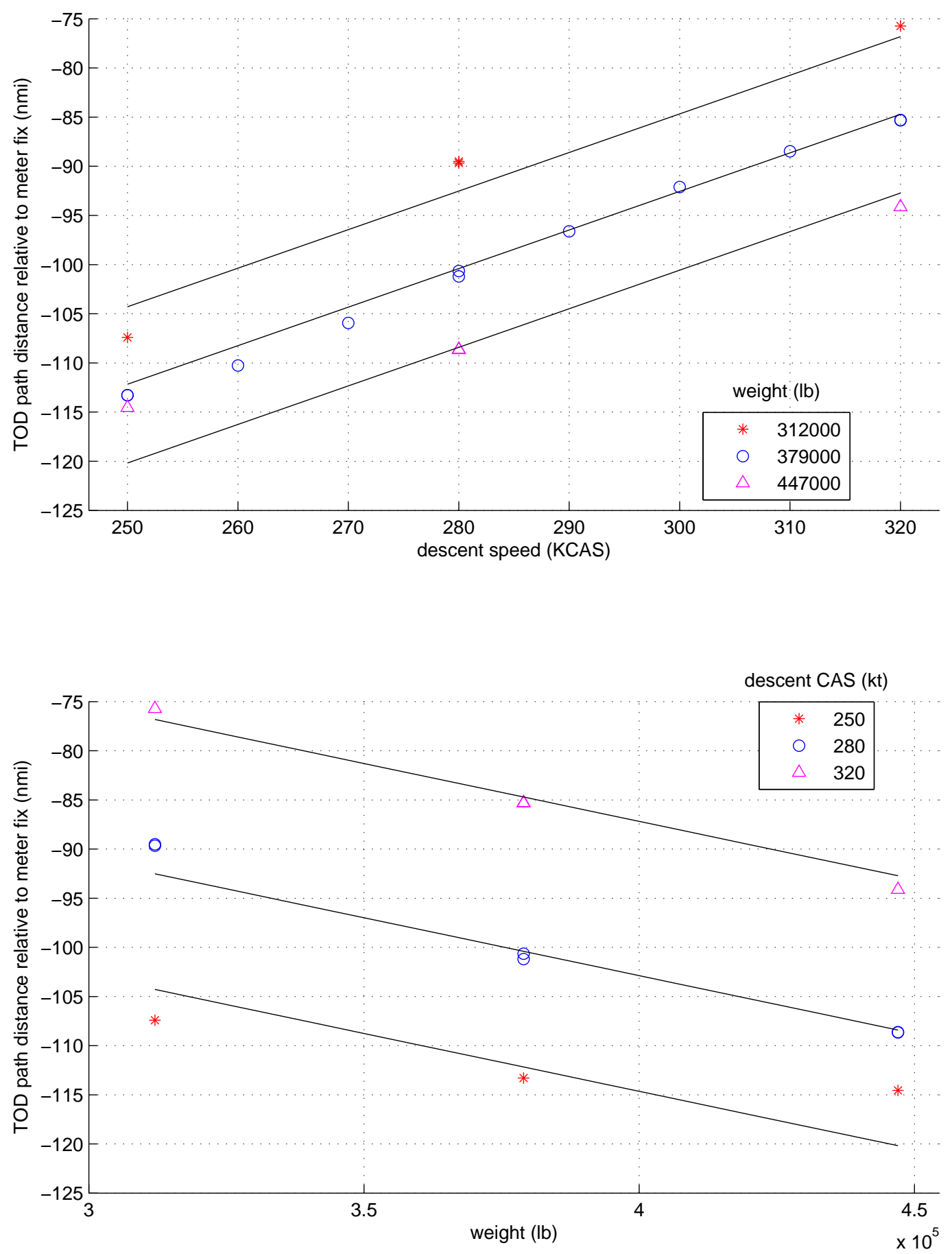

Figure 7. Speed, Weight Effect on B777 EDA TOD 


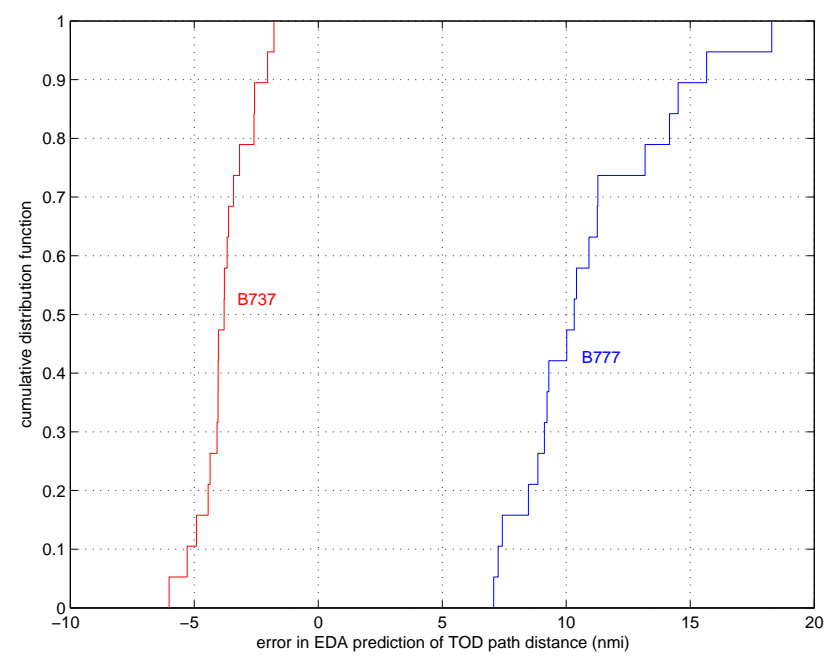

Figure 8. Difference Between FMS and EDA TOD

\section{Effect of Wind}

Figure 9 is the analogue for the B777 of Figure 3. For the six simulator runs in Subset $\mathrm{C}$ of Table 3 with the same winds used by the FMS and the simulator, which are represented by the circles in Figure 9, the TOD path distance is again linear in wind speed, with a 25-kt increase in wind speed moving the TOD location about $5.8 \mathrm{nmi}$ farther from the meter fix.

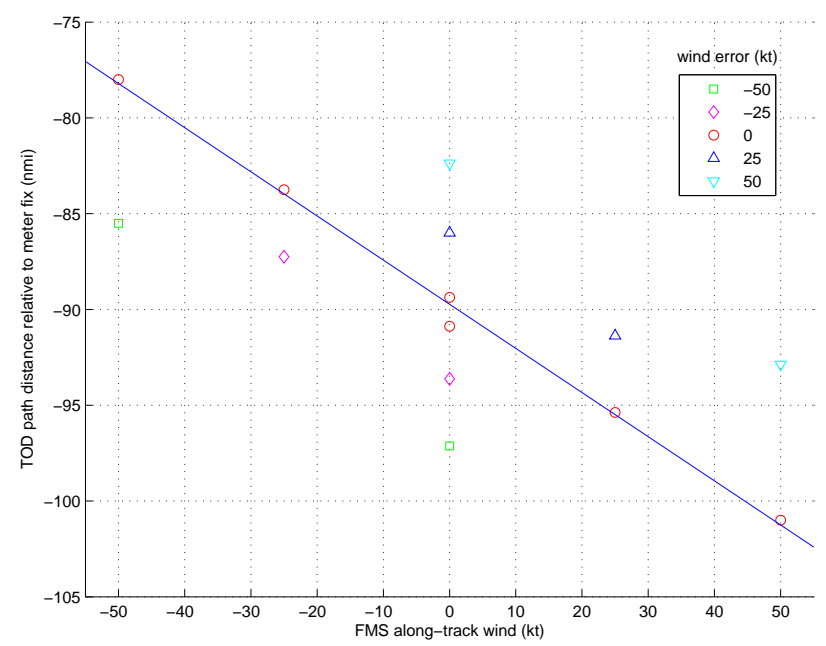

Figure 9. Effect of Wind on B777 FMS TOD

Now consider the runs in Subset $\mathrm{C}$ of Table 3 in which the simulated winds were different than those entered into the FMS descent wind page. These are indicated by the markers other than the circles in Figure 9. The error in the winds entered into the FMS descent wind page seems to have a larger effect than for the B737. Furthermore, this wind error is strongly correlated with the FMS-computed time between TOD and the meter fix, which varies over the range 780 to $861 \mathrm{sec}$ when it should be constant as explained above for the B737. This raises questions about how the B777 FMS is predicting time to fly the trajectory.

\section{Meter Fix Crossing Time}

Initial transients in the B777 simulator winds had a significant impact on the FMS predictions. For the runs in Table $3, D_{f}$ cannot be larger than $105 \mathrm{nmi}$ when analyzing the FMS estimates. Using this value, Figure 10 and Table 5 show that an approximation linear in weight and quadratic in descent speed gives a good fit of $T_{f}$. Again, adding the interaction term has little effect. For each 10,000 lb increase in weight, $T_{f}$ is about $1.0 \mathrm{sec}$ longer. If the values used in these experiments covers the operational range of weight, then using a nominal value in the middle of this range would cause errors less than $0.5 \times 0.00010 \times(447000-312000)=$ $7 \mathrm{sec}$. Whether this is acceptable would depend upon the error due to other sources.

The EDA predictions of TOD location for the B777 are significantly farther from the meter fix than the FMS-computed TOD locations, so $D_{f}$ needs to be $115 \mathrm{nmi}$ when analyzing the EDA predictions. The analogous plot to Figure 10 for the EDA predictions looks similar, although the variation due to aircraft weight is noticeably smaller. Values of $T_{f}$ based on different path lengths $D_{f}$ cannot be compared. Fortunately, $D_{f}$ can be increased to $115 \mathrm{nmi}$ for the FMS estimates for the runs in Table 2 because they started farther from the meter fix and the initial transients in the simulator winds had ended by this point. The resulting differences in $T_{f}$ are strongly correlated with descent speed. EDA gives $T_{f}$ about $35 \mathrm{sec}$ longer than does the FMS for descent speed $250 \mathrm{KCAS}, 15 \mathrm{sec}$ longer for $280 \mathrm{KCAS}$, and $5 \mathrm{sec}$ shorter for $320 \mathrm{KCAS}$. The reason is unknown.

\section{Effect of Wind}

Figure 11 is the analogue for the B777 of Figure 5. For the six simulator runs with nominal weight and descent speed and with the same winds used by the FMS and the simulator, which are represented by the circles in Figure 11, $T_{f}$ is again roughly linear in wind speed. The least squares fit line shown by the solid line indi- 

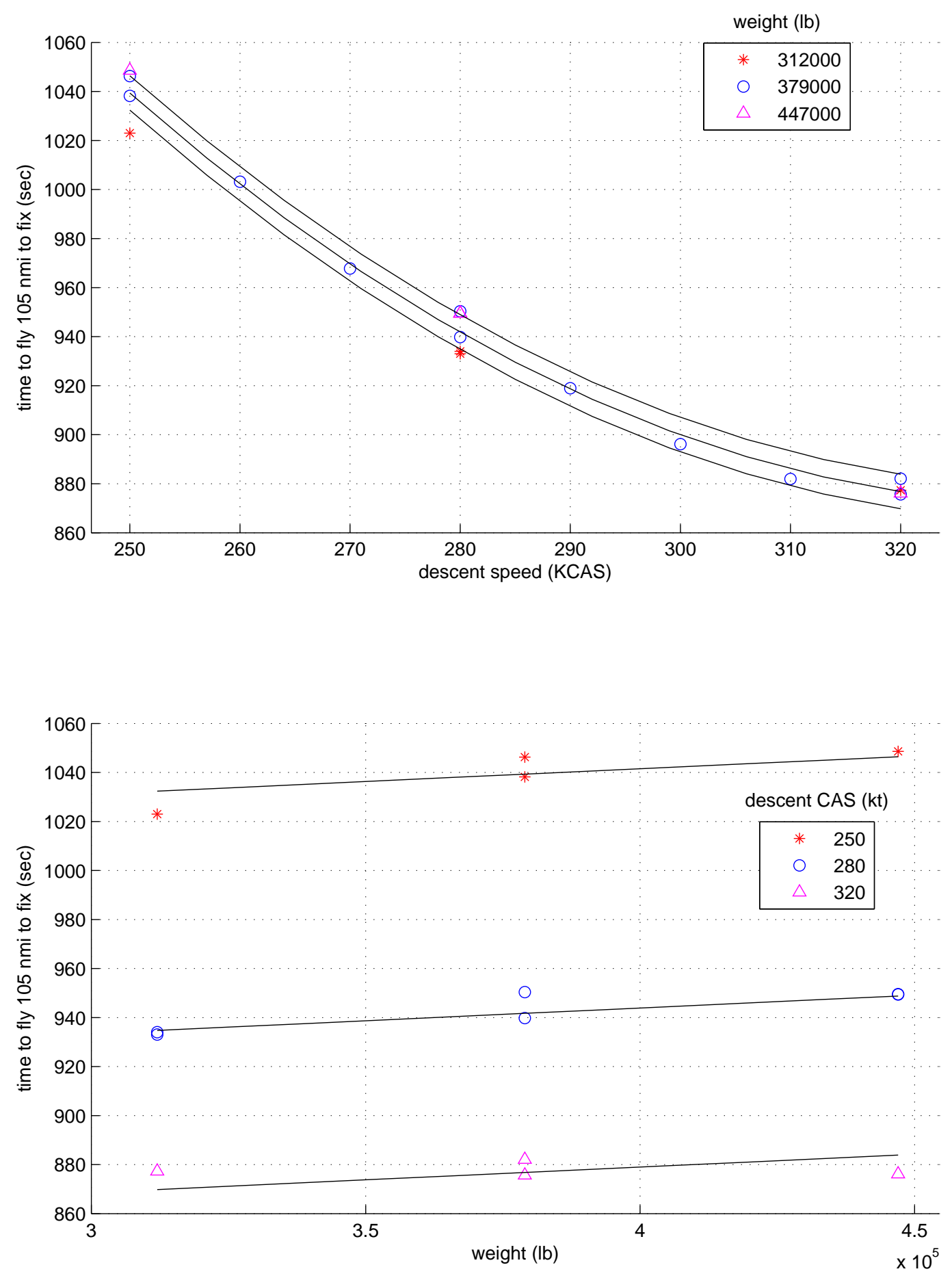

Figure 10. Speed, Weight Effect on B777 FMS Fix Time 


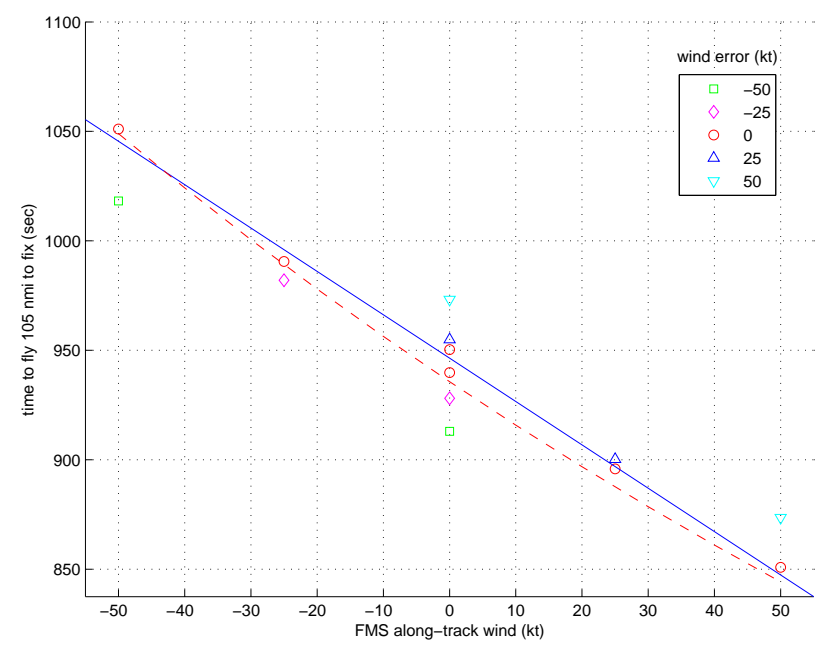

Figure 11. Effect of Wind on B777 FMS Fix Time

cates that a 25-kt increase in wind speed decreasing $T_{f}$ by about $50 \mathrm{sec}$. The dashed line shows the accuracy of equation (3), where for these descents $K$ is $820 \mathrm{sec}$ from the above discussion of the effect of wind on TOD location, $S_{\mathrm{TOD}}^{(0)}$ is $-90 \mathrm{nmi}$ and $\alpha$ is $-0.23 \mathrm{hr}$ from Fig-

ure 9, and a plot of ground speed for descents with zero wind and nominal weight shows that $\hat{v}_{c}^{(0)}$ is $467 \mathrm{kt}$. Comparison with EDA predictions for these six runs is not possible due to the necessary difference in $D_{f}$.

The markers other than the circles in Figure 11 indicate the runs in Subset C of Table 3 in which the simulated winds were different than those entered into the FMS descent wind page. As with the TOD location, the effect of this error is not quantified, but comparison with Figure 9 indicates that its relative effect on $T_{f}$ is somewhat less than its relative effect on TOD location.

\section{Conclusions}

The B737 TOD path distance is roughly linear in descent speed, aircraft weight, and wind speed. The meter fix crossing time is roughly linear in aircraft weight and wind speed. The slopes of these lines and the accuracy requirement for the predictor will determine the allowable error in the inputs used by EDA. To meet the current target of error in TOD location less than $5 \mathrm{nmi}$, EDA will need accurate values of descent speed, aircraft weight, and wind speed. To meet the current target of error in meter fix crossing time less than $20 \mathrm{sec}$, EDA will need accurate values of descent speed and wind speed but weight might not be required. The results concerning the effect of wind can be generalized to non-constant wind fields [9].

Even with perfect inputs, the EDA predictions of TOD location for the B737 are 2 to $6 \mathrm{nmi}$ from the FMS-computed locations. The cause of this will likely need to be identified and reduced in order to enable optimized descents in congested airspace. The fact that the EDA and FMS predictions of meter fix crossing time agree well for the B737 increases confidence that both are using similar equations.

For the B777, the FMS-computed TOD path distance is also roughly linear in descent speed and aircraft weight, and using a nominal value for either of these would result in absolute error in TOD location exceeding $5 \mathrm{nmi}$. The error in the EDA predictions of TOD location is 7 to $18 \mathrm{nmi}$, and the EDA prediction of TOD path distance is not linear in descent speed and aircraft weight. As with the B737, the most likely cause of this is differences between the EDA and FMS models of thrust and drag. Furthermore, the differences between the EDA and FMS estimates of meter fix crossing time indicate that at least one of them is not sufficiently accurate. EDA might be the one in error, especially given the unexpected shape of TOD location as a function of descent speed and weight. On the other hand, the B777 FMS estimates of time are under suspicion since the FMS-computed times between TOD and the meter fix depend upon wind conditions when theoretical analysis of the equations of motion show they should not.

These experiments only used two aircraft types. Stell [10] analyzed operational data for the Airbus 320 family and the B757. To keep absolute error in TOD location less than $5 \mathrm{nmi}$, the ground predictor must use accurate values of descent speed and weight for all four of these aircraft types, although nominal weight might be acceptable for the B757. Even with these inputs, the EDA predictions of TOD location are probably not sufficiently accurate for any of these aircraft types to fully enable the 3DPAM concept. Therefore, either the ground predictor must be improved or the concept must be modified. Either approach will require considerably more data - both laboratory and operational to quantify the prediction errors and validate the concept.

\section{References}

[1] Scharl, Julien, Matthew Berge, Michael L. Coats, Aslaug Haraldsdottir, Ewald G. Schoemig, 2007, 
"Modeling and Analysis of the 3D Path Arrival Management Concept," AIAA Modeling and Simulation Technologies Conference, Hilton Head, SC.

[2] Coppenbarger, Richard A., Rob W. Mead, Douglas N. Sweet, 2007, "Field Evaluation of the Tailored Arrivals Concept for Datalink-enabled Continuous Descent Approach," AIAA 5th Aviation, Technology, Integration, and Operations Conference, Belfast, Northern Ireland.

[3] Tong, Kwok-On, Daniel A. Boyle, Anthony W. Warren, 2006, "Development of Continuous Descent Arrival (CDA) Procedures for Dual-runway Operations at Houston Intercontinental," AIAA 6th Aviation, Technology, Integration, and Operations Conference, Wichita, KS.

[4] Ren, Liling, John-Paul B. Clarke, 2008, "Flight-test Evaluation of the Tool for Analysis of Separation and Throughput," Journal of Aircraft, 45, pp. 323-332.

[5] Roberts, Craig, 2007, Australian Tailored Arrivals Trial: Phase 2A Report.

[6] Wichman, Keith D., Göran Carlsson, Lars G.V. Lindberg, 2002, "Flight Trials: 'Runway-to-runway' Required Time of Arrival Evaluations for Time-based
ATM Environment," AIAA Guidance, Navigation and Control Conference, Monterey, CA.

[7] Herndon, Albert A., Ralf H. Mayer, Randal C. Ottobre, Gregory F. Tennille, 2006, Analysis of Advanced Flight Management Systems (FMSs): FMC Field Observations Trials, Technical Report MP 06W0000137, The MITRE Corporation.

[8] Herndon, Albert A., Mike Cramer, Kevin Sprong, Ralf H. Mayer, 2007, "Analysis of Advanced Flight Management Systems (FMS), Flight Management Computer (FMC) Field Observations Trials, Vertical Path," 26th Digital Avionics Systems Conference (IEEE DASC 07), Dallas, TX.

[9] Stell, Laurel L., 2009, "Flight Management System Prediction and Execution of Idle-thrust Descents," 28th Digital Avionics Systems Conference, Orlando, FL.

[10] Stell, Laurel L., 2010, "Predictability of Top of Descent Location for Operational Idle-thrust Descents," AIAA 10th Aviation, Technology, Integration, and Operations Conference, Fort Worth, TX.

29th Digital Avionics Systems Conference October 3-7, 2010 